\title{
Propuesta de seguimiento a actividades prácticas en un curso presencial a nivel superior
}

\section{Proposal for follow-up to practical activities in a higher-level presential course.}

\author{
SÁNCHEZ-LÓPEZ, Guillermina†*, ZACARÍAS-FLORES, José Dionicio y BELTRÁN-MARTÍNEZ, \\ Ramón
}

Universidad Tecnológica de Puebla, División Energías Renovables- Benemérita Universidad Autónoma de Puebla, Facultad de Ciencias Físico Matemáticas.

ID $1^{\text {er }}$ Autor: Guillermina, Sánchez-López / ORC ID: 0000-0001-5866-9362, Researcher ID: S-6946-2018, CVU CONACYT ID: 626579

ID $1^{\text {er }}$ Coautor: José Dionicio, Zacarías-Flores / ORC ID: 0000-0001-5866-9362, Researcher ID: S-7043-2018, CVU CONACYT ID: 73177

ID $2^{\text {do }}$ Coautor: Ramón, Beltrán-Martínez / ORC ID: 0000-0003--3024-4316

DOI: $10.35429 /$ JET.2019.10.3.9.16

Recibido: 10 de Octubre, 2019; Aceptado 19 de Diciembre, 2019

\begin{abstract}
Resumen
Actualmente se requiere que los egresados de nivel superior tengan conocimientos fuertes de estadística, que les permita hacer frente a las necesidades cotidianas de manejo e interpretación de información. Lo anterior no es solo una necesidad del estado de Puebla o de nuestro país, por el contrario, países como España, Estados Unidos o Inglaterra, también manifiestan un interés en el aprendizaje significativo de la estadística, lo cual puede ser observado en las diferentes planeaciones didácticas propuestas para su enseñanza, con el objetivo de que los estudiantes logren un "sentido estadístico", como lo plantean diversos autores, los cuales sugieren que para lograrlo, deben tomarse en cuenta, los siguientes puntos: desarrollar el pensamiento estadístico, usar datos reales, fomentar aprendizaje activo en el aula, promover la comprensión conceptual más que la mecanización y Agilizar el proceso de evaluación, de tal forma que se convierta en una herramienta de mejora continua.
\end{abstract}

Estadística, Proyectos, Seguimiento, Procesos, Evaluación

\begin{abstract}
At present, it is required that graduates of higher level have a strong knowledge of statistics, which allows them to face the daily needs of management and interpretation of information. The above is not only a need of the state of Puebla or our country, on the contrary, countries such as Spain, the United States or England, also show an interest in the significant learning of statistics, which can be observed in the different planning didactic proposals for teaching, with the aim of students achieve a "statistical sense", as proposed by various authors, which suggest that to achieve this, the following points should be taken into account: develop statistical thinking, use real data , encourage active learning in the classroom, promote conceptual understanding more than mechanization and streamline the evaluation process, in such a way that it becomes a tool for continuous improvement.
\end{abstract}

Statistics, Projects, Monitoring, Processes, Evaluation

Citación: SÁNCHEZ-LÓPEZ, Guillermina, ZACARÍAS-FLORES, José Dionicio y BELTRÁN-MARTÍNEZ, Ramón. Propuesta de seguimiento a actividades prácticas en un curso presencial a nivel superior. Revista Teoría Educativa. 2019. 310: $9-16$.

\footnotetext{
* Correspondencia del Autor (Correo electrónico: guille.sanlopez@ gmail.com)

$\dagger$ Investigador contribuyendo como primer autor.
} 


\section{Introducción}

Como es mencionado por Van den Bergh et al., (2006), el aprendizaje se puede ver como un proceso acumulativo, auto-regulado dirigido, colaborativo e individual, siendo importante destacar que la aplicación real del conocimiento permite al estudiante apropiarse y darle significado a lo estudiado, lo cual provocará que, al insertarse al terreno laboral, tenga conocimientos bien cimentados. Actualmente requerimos que los egresados de nivel superior tengan conocimientos fuertes de estadística, que les permita hacer frente a las necesidades cotidianas de manejo e interpretación de información, esto para cualquier área profesional.

Lo anterior no es solo una necesidad del estado de Puebla o de nuestro país, por el contrario, países como España, Estados Unidos o Inglaterra, también manifiestan un interés en el aprendizaje significativo de la estadística, lo cual puede ser observado en las diferentes planeaciones didácticas propuestas para su enseñanza, con el objetivo de que los estudiantes logren un "sentido estadístico", como lo plantean Batanero, Díaz, Contreras y Roa (2013). Franklin y Garfield (2006), citados en Zapata, (2011), los cuales sugieren que para lograrlo, deben tomarse en cuenta, los siguientes puntos:

- Desarrollar el pensamiento estadístico.

- Usar datos reales.

- Fomentar aprendizaje activo en el aula.

- Promover la comprensión conceptual más que la mecanización.

- Agilizar el proceso de evaluación, de tal forma que se convierta en una herramienta de mejora continua.

\section{Justificación}

Como sabemos dentro de cada sesión de clase deben fomentarse ciertas funciones didácticas que promuevan un aprendizaje significativo para los estudiantes, entre las cuales están: ambiente agradable, orientación de la atención, recapitulación, procesamiento de la información, interdependencia social positiva, evaluación y reflexión. (Ferreiro, 2009).
Estas funciones didácticas, están acordes a lo que en la facultad de ciencias Físico Matemáticas, se requiere lograr en los estudiantes

Debido a lo cual, desde hace algunos años se ha tomado como metodología de aprendizaje- enseñanza para los cursos de estadística en la facultad de Físico matemáticas, el aprendizaje basado en proyectos, (ABP) del cual tenemos que en diversas publicaciones se habla de lo relevante que resulta su aplicación, como generador tanto de aprendizaje significativo, como del desarrollo de competencias. Franklin et al. (2006), Rodríguez, Vargas y Luna, (2010), Batanero et al. (2013) y Sánchez (2013)

Tras la reiterativa aplicación del aprendizaje basado en proyectos, se han detectado algunos inconvenientes los cuales se ha tratado de solventar, entre los cuales está:

- La implementación del aprendizaje basado en proyectos para la enseñanza de la estadística, a nivel superior si bien, resulta ser una buena estrategia de aprendizaje - enseñanza, también es una actividad que exige dedicación y voluntad tanto del docente como de los alumnos para lograr desarrollar y culminar satisfactoriamente el proyecto en cuestión, requiere de un seguimiento muy cercano. (Rodríguez, Vargas y Luna, 2010).

- En el desarrollo del proyecto es posible "perder" el rumbo y alejarse del propósito de estudio tanto de la asignatura, como del proyecto en cuestión.

- Al llevar a cabo proyectos, los estudiantes no siempre tienen las competencias transversales desarrolladas, como son: el trabajo colaborativo, comunicación eficaz, orientación a resultados, capacidad de gestión y planificación, resolución de problemas, comunicación eficaz, tolerancia al cambio, iniciativa y proactividad, creatividad, liderazgo y aprendizaje continuo, García, Sánchez y Gavalda, (2007), lo cual es una labor más a realizar por el docente, al interior de las asignaturas. 


\section{Problemática}

La instauración del Aprendizaje basado en Proyectos, en los cursos presenciales a nivel superior, no es labor sencilla, se presentan algunas adversidades durante su aplicación, tanto en los docentes, como en los estudiantes, por lo que es necesario dar una solución efectiva a estos impedimentos, de manera que se alcance el propósito de promover en los egresados una cultura estadística y al mismo tiempo lograr el desarrollo de sus habilidades, actitudes y valores, mismos que les darán fortaleza como profesionistas.

Es por tanto imperioso que el docente tenga presente en su secuencia didáctica el dar respuesta a las siguientes preguntas.

¿Cómo evidenciar los logros de aprendizaje alcanzados por los estudiantes?

¿Cómo organizar las situaciones didácticas que se presentarán en el aula, con el propósito de hacer eficiente su aplicación?

¿Cómo lograr los objetivos de instrucción?

¿Cómo seleccionar los proyectos más viables de realizar?

¿Cómo evaluar los proyectos elaborados por los alumnos de principio a fin?

¿Cómo valorar el cumplimiento en las actividades realizadas por los equipos, así como el de cada integrante durante la elaboración de los proyectos?

¿En cuántas etapas se debe dividir la metodología para facilitar su aplicación?

¿Cómo plantear la estrategia de mejora de manera que los estudiantes más retrasados puedan tener la oportunidad de recuperarse a tiempo?

Todo lo anterior permite establecer como propósito del presente trabajo:

\section{Objetivo}

Robustecer la metodología del aprendizaje basado en proyectos, ABP, proponiendo herramientas didácticas que permitan enriquecer los procedimientos ya establecidos en la implementación de la metodología a nivel superior.

\section{Marco teórico}

Nuestra propuesta está fundamentada en la teoría constructivista, dentro del modelo colaborativo $\mathrm{y}$ en el método: Aprendizaje Basado en Proyectos ABP. De la teoría: La Construcción del aprendizaje se basa en una serie de preguntas que el docente va formulando a los estudiantes, conforme se va llevando a cabo la sesión o sesiones de clase, teniendo como objetivo que ellos sean agentes de su formación, mediante la observación, investigación y práctica de los conocimientos impartidos, lo cual será enriquecido por el trabajo colaborativo de los grupos de trabajo.

Para cumplir con lo anterior es necesario que el docente: defina claramente los objetivos de la actividad a realizar, contextualice la problemática a resolver. retroalimente las dudas generadas en los alumnos, pero sin resolver el problema y promueva la redacción completa tanto del procedimiento como de la solución del problema. Dentro de este proceso, el docente actúa como facilitador del aprendizaje, en tanto, que el alumno tiene una participación activa, construyendo su conocimiento.

\section{Del Método:}

Batanero et al. (2013) y Sánchez (2013), plantean que una buena forma de promover el sentido estadístico en los estudiantes es mediante proyectos que sean realizados por ellos.

El retomar un curso mediante esta metodología, va más allá de que los estudiantes "hagan un trabajo en equipo o una investigación y la entreguen en una fecha determinada", el llevar una asignatura mediante aprendizaje basado en proyectos, implica ir "tejiendo" una red de actividades "secuenciadas" que se van relacionando entre sí y como resultado de su aplicación y en la búsqueda de su resolución, se desprende la "necesidad de estudiar el contenido teórico de la asignatura en cuestión". 
De manera que la selección de las evidencias con las que el docente determinará el grado de desarrollo por parte de los estudiantes, resulta ser un proceso importante que debe llevarse a cabo recordando las funciones didácticas básicas a realizarse dentro de una sesión de clase, donde se debe procurar crear un ambiente agradable, que permita tanto reorientar la atención de los estudiantes hacia el trabajo en sí, como promover una Interdependencia social positiva entre los integrantes del grupo, de manera que se genere tolerancia y el respeto a las ideas presentadas por los demás, así como también que exista la retroalimentación oportuna, con la finalidad de lograr un buen procesamiento de la información.

Para lograr lo anterior los instrumentos de evaluación son la herramienta fundamental, que ayudará a determinar oportunamente el nivel de apropiación del contenido temático que tienen los estudiantes y por lo tanto establecer las estrategias de mejora para el proceso educativo.

Selección de evidencias.

El Cúmulo de acciones que realizan los estudiantes para alcanzar un propósito preestablecido en cada asignatura, permiten reforzar la aplicación significativa de conocimientos, por lo que la elección de estas, es una tarea que debe ser realizada con todo cuidado, considerando los siguientes factores:

Las evidencias deben ser:

Adaptadas al perfil del grupo en cuestión, a sus intereses, a las competencias que se quieren alcanzar. asignatura

Congruentes a los propósitos de la

Fácilmente evaluables

\section{Motivadoras}

Realizables, es decir, que con los recursos existentes pueden cumplirse en tiempo $\mathrm{y}$ forma.

Es importante también recordar que en el desarrollo de las asignaturas deben existir actividades que cumplan con:
Detectar el bagaje de conocimientos previos con que cuentan los estudiantes

Facilitar la asimilación y afianzamiento de los conocimientos

Consolidar la asimilación y aplicación de los conocimientos

\section{Reforzar el conocimiento aprendido}

Profundizar en el desarrollo de competencias transversales.

El qué y el cómo de la evaluación.

El proceso mediante el cual el docente concluye sobre el nivel de logro alcanzado por los estudiantes, tanto en conocimientos como en competencias es la llamada evaluación, la cual permite determinar la información sobre fortalezas y debilidades que los alumnos tienen, y de ser efectuada a tiempo, ayuda en la detección de riesgos de reprobación o abandono escolar y por tanto en una oportuna implementación de acciones remediales hacia los estudiantes. (Leyva 2010 y Covacevich, 2014).

$\mathrm{Al}$ interior de todas las asignaturas es necesario que el docente tenga claro: ¿Qué evaluar? y ¿Para qué evaluar?

La primera pregunta está relacionada con los propósitos que tiene la asignatura en cuestión, en tanto que para la segunda se debe determinar qué estrategias se implementarán con los resultados obtenidos.

La correcta respuesta a las preguntas anteriores permite la vinculación con la utilidad de los resultados que pueden obtenerse.

Es importante recordar que la evaluación es: sumativa, formativa y diagnóstica y cada una de estas tiene instrumentos diferentes con que realizarse. (Leyva, 2010).

Como se mencionó en párrafos anteriores debe tomarse en cuenta el objetivo específico que cada actividad puede tener, con la finalidad de determinar el instrumento más apropiado para cada una, ya que una evidencia de desempeño se valorará diferente a una de conocimiento. 
Es relevante emplear la evaluación para mejorar y valorar el aprendizaje de los estudiantes, para lo cual deben considerarse las diferentes evidencias de evaluación:

Los exámenes, tareas, proyectos, reportes escritos, presentaciones orales y además, las cuales serán valoradas con los instrumentos de evaluación correspondientes, para esto debe tomarse en cuenta:

Incorporar la evaluación como elemento sustancial del curso.

Utilizar una diversidad de métodos evaluativos que permitan brindar una evaluación completa sobre el aprendizaje de los estudiantes.

Apoyarse de la comprensión y análisis sobre artículos y gráficos presentes en los medios de información, para valorar la cultura estadística.

Evaluar el pensamiento estadístico a través de tareas de carácter de investigación. Gómez, (2017).

\section{Metodología}

El presente es un estudio cualitativo que pretende determinar las problemáticas principales que el docente pueda tener al implementar como estrategia de enseñanzaaprendizaje, el ABP. Por lo que se plantean las siguientes preguntas de investigación.

¿Cómo evidenciar los logros de aprendizaje alcanzados por los estudiantes?

¿Cómo organizar las situaciones didácticas que se presentarán en el aula, con el propósito de hacer eficiente su aplicación?

¿Cómo lograr los objetivos de instrucción?

¿Cómo seleccionar los proyectos más viables de realizar?

¿Cómo evaluar los proyectos elaborados por los alumnos de principio a fin?

¿Cómo valorar el cumplimiento en las actividades realizadas por los equipos, así como el de cada integrante durante la elaboración de los proyectos?
¿En cuántas etapas se debe dividir la metodología para facilitar su aplicación?

¿Cómo plantear la estrategia de mejora de manera que los estudiantes mas retrasados puedan tener la oportunidad de recuperarse a tiempo?

Todo lo anterior permite establecer como propósito del presente trabajo

Al término de todo curso, surge la duda, de si los contenidos teóricos que el programa de estudios marca, fueron asimilados completamente por todos los estudiantes, desafortunadamente en ocasiones es muy tarde para poder recuperar a los estudiantes que a lo largo del curso no lograron alcanzar la apropiación de conocimientos y quedan adeudando la materia o bien egresan de la carrera sin poder realizar el proceso de Meta cognición, es decir, la aplicación de lo aprendido.

Debido a lo anterior un proceso importante para el curso de estadística es la comprobación de que los estudiantes efectivamente aprendieron y aplicaron los contenidos teóricos del programa de estudio.

Para cumplir con lo anterior se inicia con la valoración de los conocimientos previos (pre test o evaluación diagnóstica) que forman el bagaje académico de los alumnos.

Este instrumento nos permitirá validar al final dicha apropiación de conocimientos.

Como siguiente actividad el docente propone al grupo la metodología que regirá el curso, es decir, el aprendizaje basado en proyectos, para lo cual se les propone que, mediante una serie de dinámicas, se realice por equipo la "detección de una necesidad a resolver".

Una vez detectada la "necesidad" se trabajará sobre la delimitación, alcances y limitantes que tenga.

Después de delimitar la "necesidad", se realizarán las investigaciones correspondientes para determinar la posible respuesta a las preguntas:

¿Qué quiero arreglar? 


\section{¿Cómo lo puedo arreglar?}

¿Para qué debe arreglarse?

Es importante considerar que esta necesidad debe ser tal que arreglarla o modificarla implique un trabajo que pueda desarrollarse en los próximos 5 meses.

La finalidad inicial de los equipos es investigar para fundamentar su "idea" de trabajo.

En la primera semana del semestre, se presentan los equipos ante el grupo para realizar la "defensa" de la "Necesidad Detectada"

Esta "ronda" consiste en elegir la mejor detección realizada por los equipos.

La necesidad mejor detectada $y$ fundamentada será la que sea abordada, para resolverla en los próximos 5 meses por cada uno de los equipos que forman el grupo.

En el desarrollo de este proceso el docente en cada clase presencial debe ir presentando una serie de preguntas que motiven en los estudiantes la investigación de conceptos relacionados con su proyecto.

De igual forma se van abordando contenidos teóricos que serán aplicados en alguna parte de los proyectos, de manera que se produce un mayor interés por parte de los integrantes del grupo, al darse cuenta que justo lo que está abordando el profesor es lo que necesitan para continuar con su trabajo, esta forma de trabajo permite que el aprendizaje se haga más significativo.

Al término del semestre se realizó un post test, instrumento que nos permitió medir el grado de apropiación del contenido teórico por parte de los estudiantes, aun cuando en el desarrollo de las etapas del proyecto puede comprobarse esto, se decidió medir por separado este logro con la finalidad de tener una Validación objetiva del ABP, cabe destacar que el post test se comparó con el pre test o prueba diagnóstica realizada en un principio.

La parte de la secuencia didáctica que se aborda en el presente trabajo podemos resumirla en el siguiente diagrama.
Diciembre, 2019 Vol.3 No.10 9-16

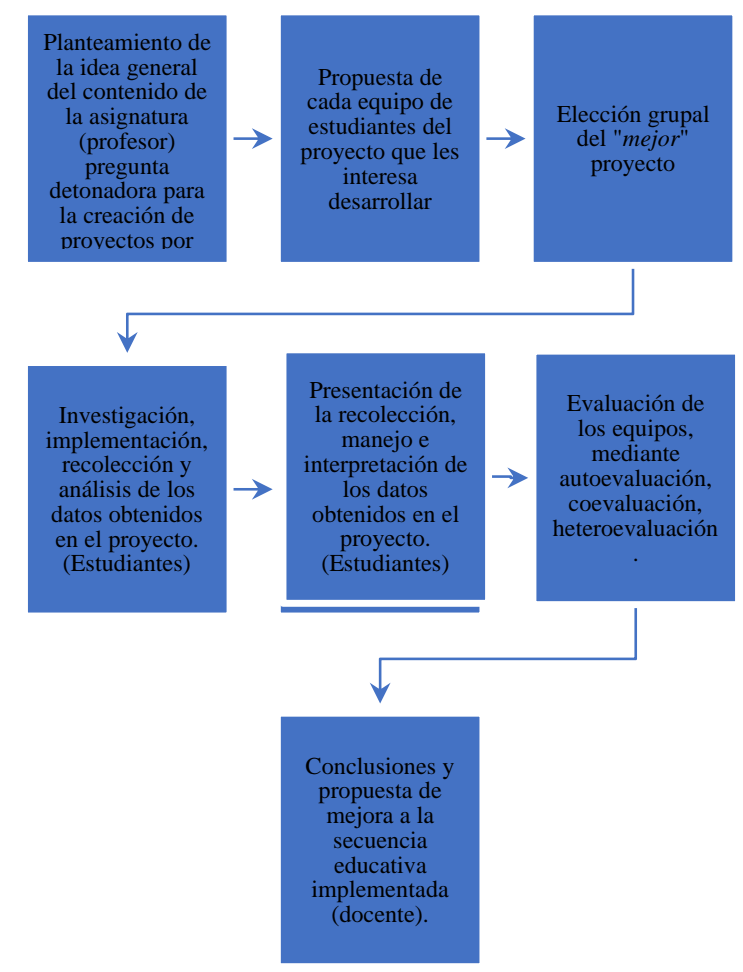

Figura 1

En la implementación del curso es necesario contar con los instrumentos de evaluación óptimos que permitan efectiva y eficazmente realizar la valoración de cada etapa, con la finalidad no solo de medir desempeño, sino de saber que está mal y por tanto dar una propuesta de cómo mejorarlo, recordando que parte de la labor docente consiste en el seguimiento oportuno a los estudiantes, de manera que pueda cumplirse el propósito de las asignaturas.

\section{Metodología de Investigación}

Con base en la experiencia obtenida tras la aplicación consecutiva de nuestra metodología, a nivel superior, tanto en la ingeniería en energías renovables de la Universidad Tecnológica de Puebla UTP, como en las licenciaturas de matemáticas, matemáticas aplicadas y actuaría de la Benemérita Universidad Autónoma de Puebla, este proyecto surge con la intención de presentar una posible manera de dar seguimiento al desarrollo de una asignatura mediante la técnica aprendizaje basado en proyectos. En todo programa de estudios a nivel superior, se tienen diversas habilidades, destrezas actitudes y aptitudes a desarrollar por parte de los estudiantes, éstas plantean en general: el cumplimiento del trabajo colaborativo, el respeto a la aportación de ideas por parte del grupo, la aplicación real del contenido temático abordado en clase haciendo uso de la tecnología preferentemente. 
Como puede observarse, éstas coinciden en que se hará una contribución al terreno laboral de profesionales capaces de enfrentarse a la solución de problemas reales, logrando trabajar en cordialidad y respeto con sus compañeros de trabajo, mostrando siempre un respeto a las instrucciones dadas por su jefe inmediato, todo lo anterior debe ser desarrollado al interior de las diferentes asignaturas que forman la currícula escolar. De manera que la instrucción actual no solo implica la transmisión de un bagaje amplio de conocimientos, si no de.

La continua implementación de la metodología en este nivel educativo ha permitido establecer no solo mejoras en la implementación, si no la determinación de la parte del experimento donde debe hacerse hincapié, y esto debido a que se está formando a ingenieros, personas que, en su desempeño profesional, deberán realizar y por tanto presentar resultados de proyectos, los cuales deben ser innovadores (Sánchez, et al, 2017).

A manera de resumen se mencionarán las mejoras que se han ido proponiendo en cada implementación. Después de la primera aplicación se concluyó que las variables donde debe hacerse mucho énfasis son: la planeación de la asignatura, el seguimiento al proyecto, el aprendizaje alcanzado por los estudiantes, la retroalimentación oportuna y la evaluación.

En la segunda aplicación se consideró que en las fases de la implementación del aprendizaje basado en proyectos que son inicio, desarrollo y final, es relevante la guía del docente para alcanzar conclusiones efectivas en los tiempos establecidos, siendo el manejo de tiempos una variable a considerar. (Sánchez, et al, 2017). Para la tercera aplicación al retomar perfil de egreso, objetivo de aprendizaje y competencia a desarrollar de la asignatura en cuestión se plantea desde cada una de las asignaturas que forman la curricula escolar de la universidad "aportar" a ese perfil de egreso, haciendo uso del diseño de experimentos como una herramienta estadística que fortalezca el desarrollo y seguimiento del proyecto hacia el emprendimiento. En este ámbito lo que se propone enfatizar en el estudiante es el emprendimiento, es decir, ¿cómo puedo hacer que mi proyecto abarque más? ¿Cuáles son las perspectivas? ¿Qué variables deben controlarse? ¿¿Cómo influyen a mi producto las variables que no se han controlado? (Sánchez, et. al, 2017)
Después de esta última implementación se concluyó que es muy importante "enseñar "al estudiante el manejo de los factores en los prototipos de manera que él sea capaz de reproducirlos. En este ámbito determinar la reproducibilidad y la replicabilidad es vital para obtener mejores y más precisos resultados.

\section{Tipo de Investigación}

La presente investigación se realiza bajo un enfoque cuantitativo, mediante un proceso deductivo que plantea inicialmente analizar el efecto que provoca en el estudiante las mejoras en la implementación del aprendizaje basado en proyectos utilizando el diseño de experimentos como herramienta de análisis de datos y de esta el seguimiento a la replicabilidad y la reproducibilidad de los prototipos.

Las Fuentes utilizadas para el proyecto se fundamentaron en las siguientes fases:

Resultados de una investigación documentada, realizada a egresados 2016, donde se mencionaba que ningún estudiante había formado una microempresa, por lo que con la finalidad de coadyuvar al emprendimiento, se da inicio a una adecuación en los planes de acción para la materia en cuestión, es importante que en el tiempo que lleva esta investigación se han ido planteando diversos objetivos, los cuales, una vez alcanzados generan una adecuación más, en un inicio solo se establece, la implementación del aprendizaje basado en proyectos en la materia de Diseño de proyectos de bioenergía, con la finalidad de inducir el emprendimiento en los estudiantes. (Sánchez, et al, 2017). Después de los datos obtenidos se determinaron y ajustaron variables por lo que se propone hacer énfasis en los factores de los prototipos a estudiar remarcando la reproducibilidad $y$ replicabilidad de cada uno, de manera que se tenga la posibilidad de mostrar al estudiante lo que significa la producción en serie.

\section{Resultados y conclusiones}

Los instrumentos utilizados los clasificamos según su uso en dos partes:

1.- Validación de apropiación de conocimientos.

2.- Validación de competencias transversales y aplicación de conocimientos 
Como se mencionó antes esta es la cuarta vez que se aplica, los resultados de cada aplicación con respecto a la medición de los instrumentos de evaluación es la siguiente:

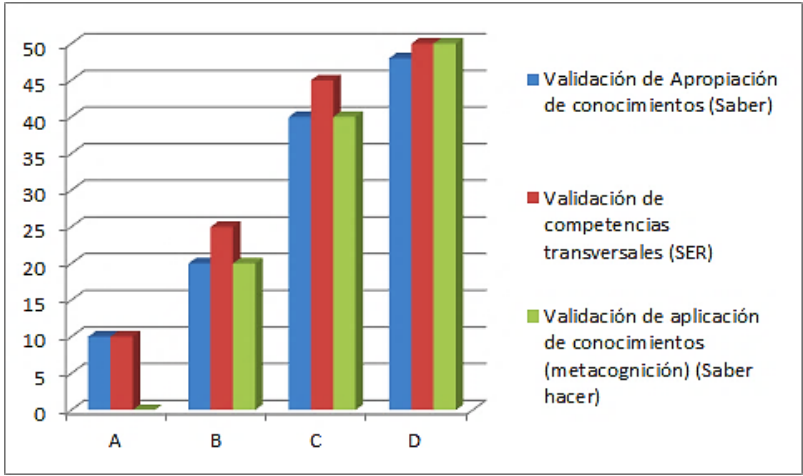

Gráfico 1

Como se observa en el Gráfico 1 el procedimiento utilizado en la implementación del ABP, permite llevar a cabo con éxito un curso donde se promueva que:

Los estudiantes partiendo de sus conocimientos previos progresivamente vayan apropiándose de los contenidos teóricos de la asignatura, hasta lograr alcanzar los propósitos planteados. Los estudiantes no solo tengan un cúmulo de conocimientos, sino también que puedan enfrentarse a retos reales aplicando lo aprendido, es decir, que tengan un aprendizaje práctico. El desarrollo de las actividades propuestas ayudan al crecimiento personal de los alumnos, al promover actitudes como la tolerancia, el trabajo colaborativo, respeto al expresar sus ideas. Estas situaciones quedaron plasmadas en la gráfico 2, de donde observamos que en cada implementación se ha podido mejorar la metodología del ABP, así como también se tiene que los instrumentos de evaluación utilizados también se han perfeccionado, resultando ser más eficientes para realizar una retroalimentación oportuna en el proceso educativo.

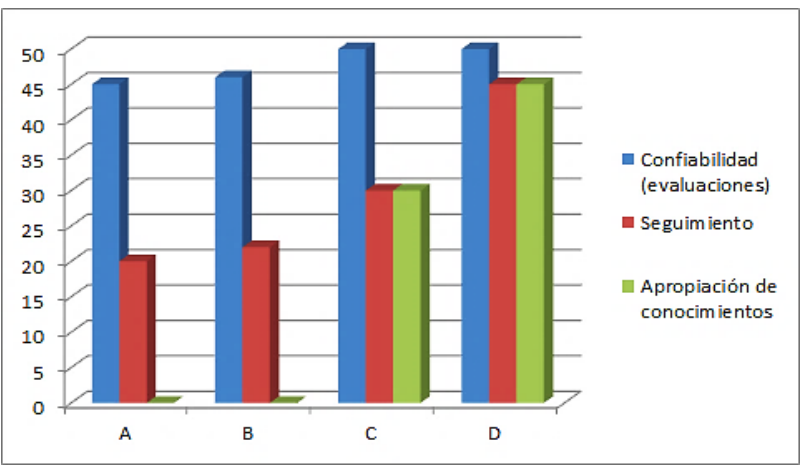

Gráfico 2

\section{Contribución}

Se ha observado que gracias a la implementación de esta metodología, los estudiantes partiendo de sus conocimientos previos progresivamente van apropiándose de los contenidos teóricos de la asignatura, hasta lograr alcanzar los propósitos planteados. Al término de los cursos se ha observado que los estudiantes no solo tienen un cúmulo de conocimientos, sino también son capaces de enfrentarse a retos reales aplicando lo aprendido, es decir, que se logra un aprendizaje práctico. Por otra parte el desarrollo de las actividades propuestas ayudan al crecimiento personal de los alumnos, al promover actitudes como la tolerancia, el trabajo colaborativo, respeto al expresar sus ideas. El presente trabajo aporta una serie de instrumentos que los docentes pueden utilizar en el seguimiento de proyectos implementados en sus diferentes asignaturas.

\section{Referencias}

Ferreiro, R, Nuevas alternativas de aprender y enseñar, aprendizaje cooperativo, México, Trillas, 2009

García García, M. J., Fernández Sanz, L., Terrón López, M. J., Blanco Arcilla, J. "Métodos de evaluación para las competencias generales más demandada en el mercado laboral", JENUI, 2008.

Gomez Arroyo Danae. (2017). Propuesta de mejora a la metodología aprendizaje basado en proyectos para la enseñanza de la estadística. Puebla, BUAP.

Van Den Bergh, V., Mortermans, D., Spooren, P., Van Petegem, P., Gijbels, Dan den bergh, V.,Mortermans, D., Spooren, P., Van Petegem, P., Gijbels, D., Vanthournout, G. New assessment modes within project-based education - the stakeholders. Studies in Educational Evaluation, Kiel, Germany, v. 32, p. 345-368, 2006. 